\title{
Bibliography of Professor Stefan Jakobielski
}

\section{BOOKS}

1972 A History of the Bishopric of Pachoras on the Basis of Coptic Inscriptions, Faras III, Warszawa

2016 Chronologia wczesnych malowideł ściennych z katedry w Faras (VIII-IX wiek), Paideia 1, Warszawa

2017 (with Martens-Czarnecka, M., Łaptaś, M., Mierzejewska, B., Rostkowska, B.) Pachoras. Faras: The wall paintings from the Cathedrals of Aetios, Paulos and Petros, PAM Monograph Series 4, Warsaw

\section{CHAPTERS, CONTRIBUTIONS IN CONGRESSES PROCEEDINGS} AND EXHIBITION CATALOGUES

1960 Rękopisy koptyjskie, [in:] Andrzejewski, T., Jakobielski, S., Strelcyn. S. (Eds), Katalog rękopisów egipskich, koptyjskich i etiopskich, Katalog rękopisów orientalnych ze zbiorów polskich IV, Warszawa, 25-42

1962 (with Marciniak, M.) Inscriptions grecques et coptes, [in:] Michałowski, K., Fouilles polonaises, Faras I, Warszawa, 109-118

1965 Inscriptions chrétiennes, [in:] Michałowski, K., Jakobielski, S., Fouilles polonaises 1961-1962, Faras II, Warszawa, 163-201

1966 Two Coptic Foundation Stones from Faras, [in:] Bernhardt, M.L. (Ed.), Mélanges offerts à Kazimierz Michałowski, Warszawa, 103-109

1970 Polish Excavations at Old Dongola 1969, [in:] Dinkler, E. (Ed.), Kunst und Geschichte Nubiens in christlicher Zeit, Recklinghausen, 171-180 
1970 Some Remarks on Faras Inscriptions, [in:] Dinkler, E. (Ed.), Kunst und Geschichte Nubiens in christlicher Zeit, Recklinghausen, 29-39

1974 Chronologia Biskupów Faras / Chronology of Bishops of Faras / Chronologie der Bischöfe von Faras, [in:] Michałowski, K., Faras, Malowidła ścienne w zbiorach Muzeum Narodowego w Warszawie / Faras, Wall Paintings in the Collection of the National Museum in Warsaw / Die Wandbilder in den Sammlungen des Nationalmuseums zu Warschau, Warszawa, 321-323 / 318-320 / 332-334

1974 Inskrypcje / Inscriptions / Inschriften, [in:] Michałowski, K., Faras, Malowidła ścienne w zbiorach Muzeum Narodowego w Warszawie / Faras, Wall Paintings in the Collection of the National Museum in Warsaw / Die Wandbilder in den Sammlungen des Nationalmuseums zu Warschau, Warszawa, 279-312 / 277-309 / 287-324

1975 Polish Excavations at Old Dongola 1970-72, [in:] Michałowski, K. (Ed.), Nubia, Récentes Recherches. Actes du colloque Nubiologique International au Musée National de Varsovie 19-22 Juin 1972, Varsovie, 70-75

1975 (with Dzierżykray-Rogalski, T.) La tombe de l'Eparque Yoannès dans l'église à Colonnes en Granit de Dongola (Soudan), [in:] Michałowski, K. (Ed.), Nubia, Récentes Recherches. Actes du colloque Nubiologique International au Musée National de Varsovie 19-22 Juin 1972, Varsovie, 44-48

1978 Polish Excavations at Old Dongola 1973 and 1974 season, [in:] Leclant, J., Vercoutter, J. (Eds), Études Nubiennes, Colloque de Chantilly 2-6 Juillet 1975, BiEtud LXXVII, Le Caire, 127-140

1978 Inscriptions from Faras and the problems of the chronology of murals, [in:] Leclant, J., Vercoutter, J. (Eds), Études Nubiennes, Colloque de Chantilly 2-6 Juillet 1975, BiEtud LXXVII, Le Caire, 141-151

1978 Chrześcijaństwo nubijskie w świetle najnowszych badań, [in:] Atiya, A.S., Historia Kościołów Wschodnich, Warszawa, 385-393

1982 Remarques sur la chronologie des peintures murales de Faras aux VIII et IX ${ }^{\mathrm{e}}$ siècles, [in:] Jakobielski, S. (Ed.), NubChrist I, Warszawa, 142-172

1982 Portraits of the Bishops of Faras, [in:] Plumley, J.M. (Ed.), Nubian Studies, Proceedings of the Symposium for Nubian Studies, Selwyn College, Cambridge, 1978, Warminster, 127-133 
1982 Polish Excavations at Old Dongola in 1976 and 1978, [in:] Plumley, J.M. (Ed.), Nubian Studies, Proceedings of the Symposium for Nubian Studies, Selwyn College, Cambridge, 1978, Warminster, 116-126

1982 A brief account on the churches of Old Dongola, [in:] Moorsel, P. van (Ed.), New discoveries in Nubia, Proceedings of the Colloquium on Nubian Studies, The Hague, 1979, Leiden, 51-56

1986 Faras, [in:] Kiss, Z. (Ed.), 50 lat polskich wykopalisk w Egipcie i na Bliskim Wschodzie, Warszawa, 85-90

1986 Stara Dongola, [in:] Kiss, Z. (Ed.), 50 lat polskich wykopalisk w Egipcie i na Bliskim Wschodzie, Warszawa, 91-96

1986 Polish Excavations at Old Dongola, 1978/79-1982, [in:] Krause, M. (Ed.), Nubische Studien. Tagungsakten der 5. Internationalen Konferenz der International Society for Nubian Studies, Heidelberg 22.-25. September 1982, Mainz a/Rhein, 299-310

1987 North and South in Christian Nubian Culture: Archaeology and History, [in:] Hägg, T. (Ed.), Nubian Culture - Past and Present. Main Papers presented at the Sixth International Conference for Nubian Studies in Uppsala, 11-16 August 1986, Kungl. Vitterhets Historie och Antikvitets Akademien - Konferenser 17, Stockholm, 231-235

1988 Christian Nubia at the height of its civilization, [in:] El Fasi, M. (Ed.), General History of Africa (UNESCO), vol. III: Africa from the Seventh to the Eleventh Century, Paris-Berkeley, 194-223

1990 (with Medeksza, S.) The North-West Church at Old Dongola, [in:] Godlewski, W. (Ed.), Coptic Studies, Acts of the Third International Congress of Coptic Studies, Warsaw, 20-25 August 1984, Warszawa, 165-174

1991 The inscriptions, ostraca and graffiti, [in:] Welsby, D.A., Daniels, C.M. (Eds), Soba, Archaeological Research at a Medieval Capital on the Blue Nile, Memoirs of the British Institute in Eastern Africa 12, London, 274-296

1995 Dongola, dzieje, archeologia, znaczenie, [in:] Bernhard, M.L. (Ed.), Od Nilu do Eufratu. Polska Archeologia Śródziemnomorska 1981-1994, Warszawa, 76-87

1995 Monastery of the Holy Trinity at Old Dongola - A Short Archaeological Report, [in:] Starowieyski, M. (Ed.), The Spirituality of Ancient Monasticism. Acts of the International Colloquium held in Cracow-Tyniec 16-19th November 1994. Specialised Contributions, Cracow-Tyniec, 35-45 
1996 The Early Christian Period in Nubia, [in:] Herrmann, J., Zürcher, E. (Eds), History of Humanity, Scientific and Cultural Development, vol. III: From the Seventh Century BC to the Seventh Century AD, UNESCO, Paris-New York, 326-331

1996 Nubia w okresie chrześcijańskim, [in:] Tymowski, M. (Ed.), Historia Afryki. Do początku XIX wieku, Wrocław-Warszawa-Kraków, 545-569

1996 (with Żurawski, B.) Kusz, [in:] Tymowski, M. (Ed.), Historia Afryki. Do początku XIX wieku, Wrocław-Warszawa-Kraków, 213-232

1998 Kościoły w Starej Dongoli, [in:] Iwaszkiewicz-Wronikowska, B. (Ed.), Sympozja Kazimierskie poświęcone kulturze świata późnego Antyku i wczesnego chrześcijaństwa, Lublin, 277-285

1998 The Monastery in Old Dongola: Excavations of the Western Annexe 1995-1997, [in:] Paner, H. (Ed.), Gdańsk Archaeological Museum African Reports 1, Gdańsk, 55-61

200135 Years of Polish Excavations at Old Dongola. A Factfile, [in:] Jakobielski, S., Scholz, P.O. (Eds), Dongola-Studien, 35 Jahre polnischer Forschungen im Zentrum des makuritischen Reiches, Bibliotheca nubica et aethiopica 7, Warszawa-Wiesbaden, 1-48

2001 Das Kloster der Heiligen Dreifaltigkeit. Bauphasen des nordwestlichen Anbaus, [in:] Jakobielski, S., Scholz, P.O. (Eds), Dongola-Studien, 35 Jahre polnischer Forschungen im Zentrum des makuritischen Reiches, Bibliotheca nubica et aethiopica 7, Warszawa-Wiesbaden, 141-168

2001 (with Łaptaś, M.) 'Unknown’ Mural of the Three Youths in a Fiery Furnace from the Faras Cathedral, [in:] Bulsza, W., Sadko, L. (Eds), Ars graeca, ars latina. Studia dedykowane Annie Różyckiej-Bryzek, Kraków, 75-85

2002 Faras, das meroitische Phrs, das mittelalterliche Pachoras, [in:] Seipel, W. (Ed.), Faras. Die Kathedrale aus dem Wüstensand, Ausstellungskatalog des Kunsthistorischen Museums, Wien, 35-36

2002 Die Baugeschichte der Kathedrale, [in:] Seipel, W. (Ed.), Faras. Die Kathedrale aus dem Wüstensand, Ausstellungskatalog des Kunsthistorischen Museums, Wien, $37-40$

2002 Die Chronologie der Wandmalereien in der Kathedrale von Faras, [in:] Seipel, W. (Ed.), Faras. Die Kathedrale aus dem Wüstensand, Ausstellungskatalog des Kunsthistorischen Museums, Wien, 51-56 
2002 Die Rettung der Wandgemälde von Faras, [in:] Seipel, W. (Ed.), Faras. Die Kathedrale aus dem Wüstensand, Ausstellungskatalog des Kunsthistorischen Museums, Wien, 57-60

2002 Polnische Ausgrabungen in Alt-Dongola, [in:] Seipel, W. (Ed.), Faras. Die Kathedrale aus dem Wüstensand, Ausstellungskatalog des Kunsthistorischen Museums, Wien, 61-62

2002 Old Dongola - Vieux Dongola - Dunqula al-Ağuz, [in:] Saving the Sudan Ancient Cultural Heritage. Forty Years of Cooperation in Archaeology between the Sudan and Poland, Poznań, 15-20

2004 catalogue entries: Wall painting of Christ Victor; The Holy Trinity; Window grille or partition; Oil lamp; Starburst lamp; Tombstone of Petros; Bowl of Lazaros; Cross, [in:] Welsby, D.A., Anderson, J.R. (Eds), Sudan. Ancient Treasures, London, 214-215

2005 The Monastery in Old Dongola: Excavation of the North-Western Annexe 19982002, [in:] Paner, H., Jakobielski, S. (Eds), Gdańsk Archaeological Museum African Reports 3, Gdańsk, 107-126

2006 Old Dongola / Stara Dongola / Dunqula al-Ağuz, [in:] Jakobielski, S. (Ed.), Polish Excavation at Old Dongola, 45 years of the archaeological cooperation with the Sudan / Polskie Wykopaliska w Starej Dongoli: 45 lat współpracy archeologicznej z Sudanem, Warszawa, 9-15

2006 Klasztor Świętej Trójcy / The Holy Trinity Monastery, [in:] Jakobielski, S. (Ed.), Polish Excavation at Old Dongola, 45 years of the archaeological cooperation with the Sudan / Polskie Wykopaliska w Starej Dongoli: 45 lat współpracy archeologicznej z Sudanem, Warszawa, 16-32

2006 catalogue entries, Polish and English version, [in:] Jakobielski, S. (Ed.), Polish Excavation at Old Dongola, 45 years of the archaeological cooperation with the Sudan / Polskie Wykopaliska w Starej Dongoli: 45 lat współpracy archeologicznej z Sudanem, Warszawa, 49-74

2008 The Holy Trinity Monastery in Old Dongola, [in:] Godlewski, W., Łajtar, A. (Eds), Between the Cataracts. Proceedings of the $11^{\text {th }}$ International Conference for Nubian Studies, Warsaw University, 27 August - 2 September 2006, PAM Supplement Series 2.1, Warsaw, 283-302 
2009 The Monastery of the Holy Trinity at Old Dongola (Sudan). Some Key Information (Opening Lecture), [in:] Popielska-Grzybowska, J., Białostocka, O., Iwaszczuk, J. (Eds), Proceedings of the Third Central European Conference of Young Egyptologists, Egypt 2004: Perspectives of Research, Warsaw 12-14 May 2004, AAP I, 73-87

2010 The Holy Trinity Monastery in Old Dongola. Excavation 2002/2003 - 2006, [in:] Paner, H., Jakobielski, S. (Eds), Gdańsk Archaeological Museum African Reports 6, Gdańsk, 69-90

2011 (with Vliet, J. van der) From Aswan to Dongola: The Epitaph of Bishop Joseph (died AD 668), [in:] Łajtar, A., Vliet, J. van der (Eds), Nubian Voices. Studies in Christian Nubian Culture, JJP-Suppl. XV, Warsaw, 15-35

2013 Chrześcijaństwo w Nubii / Christianity in Nubia, [in:] Bagińska, D. (Ed.), Wielkie Królestwa chrześcijańskie w Nubii / The Great Christian Kingdoms of Nubia, Poznań, 5-14

2013 Faras, [in:] Bagińska, D. (Ed.), Wielkie Królestwa chrześcijańskie w Nubii / The Great Christian Kingdoms of Nubia, Poznań, 15-20

2013 Katedra w Faras / Faras Cathedral, [in:] Bagińska, D. (Ed.), Wielkie Królestwa chrześcijańskie w Nubii / The Great Christian Kingdoms of Nubia, Poznań, 20-29

2013 Średniowieczne malarstwo nubijskie / Medieval Nubian Painting, [in:] Bagińska, D. (Ed.), Wielkie Królestwa chrześcijańskie w Nubii / The Great Christian Kingdoms of Nubia, Poznań, 31-40

2013 Stara Dongola / Old Dongola, [in:] Bagińska, D. (Ed.), Wielkie Królestwa chrześcijańskie w Nubii / The Great Christian Kingdoms of Nubia, Poznań, 41-48

2013 Klasztor Świętej Trójcy / The Holy Trinity Monastery, [in:] Bagińska, D. (Ed.), Wielkie Królestwa chrześcijańskie w Nubii / The Great Christian Kingdoms of Nubia, Poznań, 49-60

2014 Malowidła z Faras. Pół wieku po odkryciu, [in:] Szafrański, S., Kądziela, M., Tobota, M., Ząbek, M. (Eds), Sztuka Afryki w kolekcjach i badaniach polskich, Szczecin, 253-280 


\section{JOURNAL PAPERS}

1966 Grecka inskrypcja fundacyjna Katedry w Faras, RMNW X, Warszawa, 99-106

1966 La liste des évêques de Pakhoras, EtudTrav I, 151-170

1968 (with Ostrasz, A.) Polish Excavations at Old Dongola - Second Season, December 1965-February 1966, Kush XV, 125-142

1968 (with Krzyżaniak, L.) Polish Excavations at Old Dongola - Third Season, December 1966-February 1967, Kush XV, 143-164

1969 Some new data to the history of Christian Nubia as found in Faras' inscriptions, Klio 51, 499-508

1971 Nouvelle lecture d'un nom de la Liste des Evêques de Pakchoras, EtudTrav V, 193-199

1975 Old Dongola 1972-1973, EtudTrav VIII, 349-360

1978 Dongola 1974, EtudTrav X, 407-416

1979 Dongola 1976, EtudTrav XI, 229-244

1981 Nubian Christian Architecture, ZÄS 108, 33-48

1981 (with Godlewski, W.) Od Faras do Dongoli, Meander 6, 351-355

1982 A rock-cut tomb in el-Ghaddar (Dongola), Meroitica 6, 175-177

1983 Coptic Graffiti from Faras, EtudTrav XIII, 133-137

1984 Documentation Project for Nubian Murals, NubLett 3, 5-10

1985 (with Medeksza, S.) The North-West Church in Old Dongola, NubLett 5, 2-3

1989 Nubiè, Agape, Interdisciplinair vroegchristelijk dispuut Jaarboek 1988-89, $13-18$

1990 Badania w Starej Dongoli w 1989 roku, Raporty Wykopaliskowe Centrum Archeologii Śródziemnomorskiej Uniwersytetu Warszawskiego I, 75-82 
1990 (with Godlewski, W.) Dongola 1978-1980, EtudTrav XIV, 393-408

1991 Old Dongola 1989-1990, PAM II, 65-73

1993 Old Dongola, 1991-1992, PAM IV, 98-110

1993 Stara Dongola 1991/92, Raporty Wykopaliskowe Centrum Archeologii Śródziemnomorskiej Uniwersytetu Warszawskiego IV, 90-105

1993 Old Dongola, Preliminary Report on the Excavations for the Directorate General of Antiquities and National Museums, Khartoum, Covering the field season of 1990 (January - February), Kush XVI, 300-304

1993 Old Dongola, The report on salvage works on Kom H effected in 1990 field season, Kush XVI, 305-309, 328-333

1993 (with Pluskota, K., Żurawski, B.) Polish Excavations at Old Dongola, twenty-fifth season 1991/92, Kush XVI, 288-299, 310-327

1994 Old Dongola 1993, PAM V, 115-128

1994 Stara Dongola 1993, Raporty Wykopaliskowe Centrum Archeologii Śródziemnomorskiej Uniwersytetu Warszawskiego V, 106-120

1995 Old Dongola 1993/94, PAM VI, 84-92

1995 Stara Dongola 1993/94, Raporty Wykopaliskowe Centrum Archeologii Śródziemnomorskiej Uniwersytetu Warszawskiego VI, 89-97

1995 Chronological Implication of G.M. Browne’s Reading of Griffith’s Old Nubian Graffito 4, EtudTrav XVII, 29-34

1996 Old Dongola, Monastery 1995, PAM VII, 103-113

1997 Old Dongola, Excavations 1996, PAM VIII, 159

1997 Old Dongola, Kom H, Site NW, PAM VIII, 161-168

1997 (with Łajtar, A.) Ein Glaubensbekenntnis aus Alt Dongola (Sudan), JJP XXVII, $7-26$

1998 Old Dongola, Excavations 1997, PAM IX, 158-159 
1998 Old Dongola, Kom H, PAM IX, 160-169

1999 Old Dongola Excavations, 1998, PAM X, 137-147

2000 Old Dongola, Documentation work in the Monastery, 1999, PAM XI, 207-208

2001 Old Dongola, Season 2000, PAM XII, 265-279

2001 Tentative d'identification de certaines peintures de Faras, EtudTrav XIX, 59-79

2002 Polnische archäologische Ausgrabungen in Nubien, Vernissage 106 (13/02), 6-15

2003 Old Dongola, Fieldwork in 2002, PAM XIV, 211-229

2005 Old Dongola, Fieldwork in 2004, PAM XVI, 259-272

2007 Nubian Scenes of Protection from Faras as an Aid to Dating, EtudTrav XXI, 43-50

2007 Archeologia Nubii. Wykopaliska Ekspedycji Polskich, Signa Temporis 14, 19-37

2008 (with Martens-Czarnecka, M.) Old Dongola. Fieldwork, Seasons 2005/2006 and 2006, PAM XVIII, 325-342

2013 Horned Crown - an Epigraphic Evidence, EtudTrav XXVI/1, 325-337

2016 The Murals on the Entrance Porch of the Faras Cathedral, EtudTrav XXIX, 75-94

\section{ENTRIES IN ENCYCLOPEDIAS}

1983 Dongola, [in:] Encyklopedia Katolicka IV, Lublin, 114-115

1989 Faras, [in:] Encyklopedia Katolicka V, Lublin, 45-46

1994 Dongola, [in:] Enciclopedia dell’Arte Antica, Supplemento II, Roma, 396-397

OTHER

1975 Observations sur un livre récemment paru : Jadwiga Kubińska, Faras IV - Inscriptions grecques chrétiennes, EtudTrav VIII, 323-332

1977 Badania archeologiczne w Starej Dongoli (Sudan), Problemy 12/381, 20-24 
1992 Excavations in the capital city of the Kingdom of Makouria, Newsletter of the Sudan Archaeological Research Society 2, 2-3

1997 Nouvelles peintures murales du désert de Nubie, Le Monde de la Bible 102, 65

1999 Obituary, Jack Plumley, 1910-1999, PAM X, Reports 1998, 11-12

2001 Bibliography of Polish Excavations at Old Dongola, [in:] Jakobielski, S., Scholz, P.O. (Eds), Dongola-Studien, 35 Jahre polnischer Forschungen im Zentrum des makuritischen Reiches, Bibliotheca nubica et aethiopica 7, Warszawa-Wiesbaden, 395-402

2002 A new book on Nubia 'Nubische Studien (Hrsgg. v. S. Jakobielski \& P.O. Scholz), Warszawa 2001', Polish Academy of Sciences Annual Report 2002

2014 Kamila Kołodziejczyk (1929-2013), EtudTrav XXVII, 13-17

2017 Życie i dziedzictwo Profesora Kazimierza Michałowskiego - świadectwo ucznia / Professor Kazimierz Michałowski’s Life and Heritage - Testimony of a Disciple / Hayāt wa-turāt ustād Kāzīmīš Mīhāāūfskī - šahādat țālibih, [in:] Jastrzębski, M.M., Szczęsny-Kostanecki, D. (Eds), Pol + Mashreq Conference: Artykuły i prelekcje o historii i przyszłości polsko-egipskiej współpracy / Pol + Mashreq Conference: Articles on the History and Future of Polish-Egyptian Cooperation, Warszawa, 19-27 / 28-43 / 44-53

2018 Treasures from Faras, Polish Academy of Sciences Annual Report 2018, 12-14 


\section{ÉTUDES et TRAVAUX XXXII / 2019}

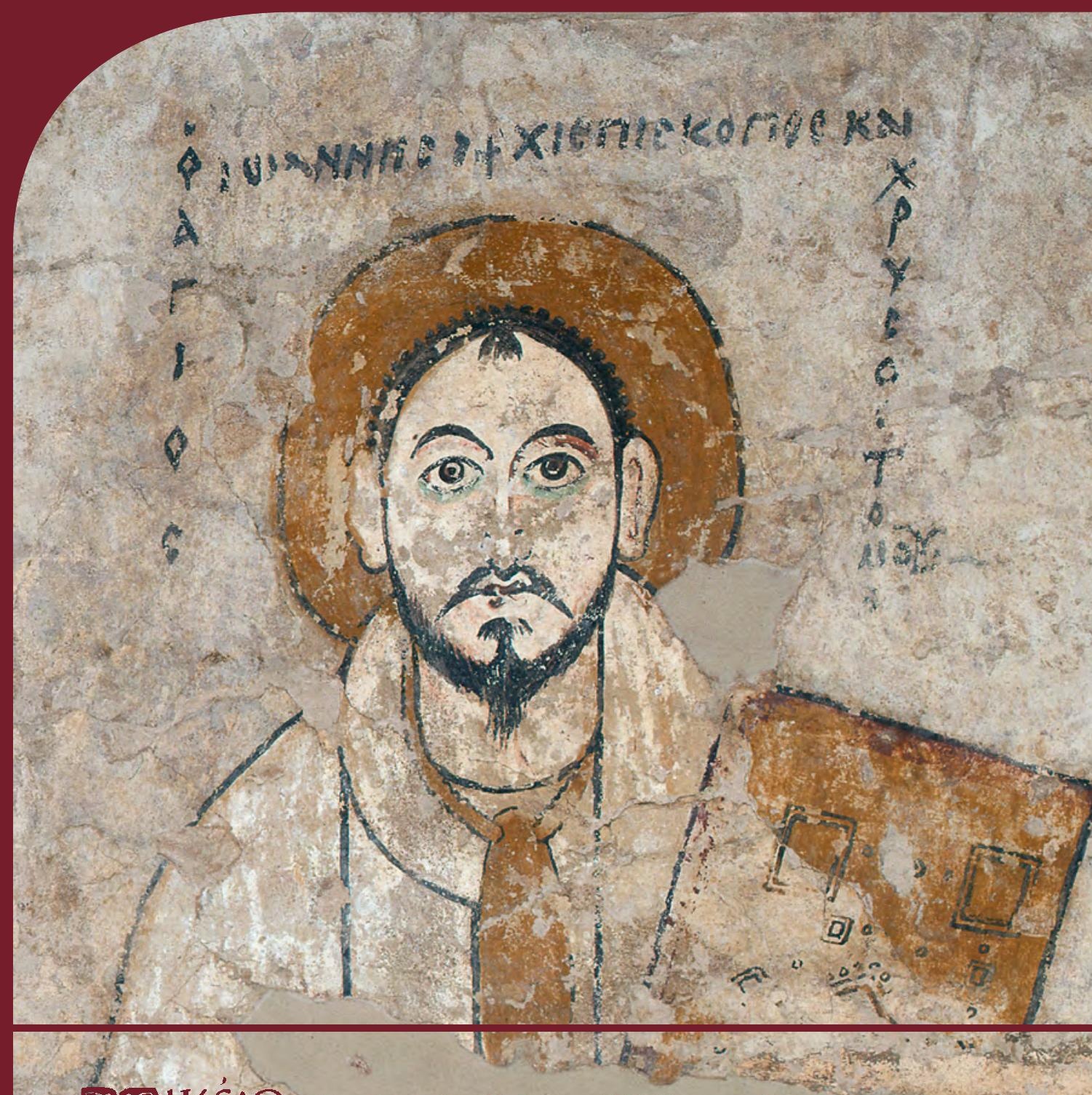

BOMIKSiO Institut des Cultures Méditerranéennes et Orientales ED2A PAN de l'Acácémie Polonaise des Sciences 


\title{
COMITÉ DE RÉDACTION SCIENTIFIQUE
}

Maciej Makowski - rédacteur en chef Jadwiga Iwaszczuk - rédacteur

Maciej G. Witkowski - rédacteur

Agnieszka Ryś - sécretaire de la rédaction

Bogdan Żurawski - rédacteur thématique du volume

CONSEIL SCIENTIFIQUE DU JOURNAL

M. Kobusiewicz (IAE PAN, Warszawa), E. Laskowska-Kusztal (IMOC PAS, Warszawa),

D. Michaelides (University of Cyprus, Nicosia),

J.Ch. Moretti (IRAA-MOM, Université de Lyon 2/CNRS),

D. Raue (Ägyptisches Museum der Universität Leipzig), P. Reynolds (ICREA, España),

D. Welsby (British Museum, London)

\section{COMITÉ SCIENTIFIQUE DE LECTURE}

D.E. Arnold (Wheaton College/The Field Museum, Chicago), H.D. Baker (University of Toronto),

P. Ballet (ArScAn-ESPRI, Université Paris Nanterre), Ch. Barber (Princeton University),

N. Beaux-Grimal (IFAO, Caire/Collège de France, Paris), A. Boud'hors (CNRS, IRHT, Paris),

J. Budka (Ludwig-Maximilians-Universität München), R. David (SFDAS),

A. Delattre (CPEG, Bruxelles), A. Dodson (University of Bristol),

E. Fogliadini (Facoltà Teologica dell'Italia Settentrionale, Milan),

V. Francigny (CNRS/CRES, Paris), L. Gabolde (CNRS), C. Gobeil (Egypt Exploration Society, London),

N. Hamdi (UCL, Louvain), J. den Heijer (CIOL/INCAL, Louvain-la-Neuve),

S. Ikram (American University in Cairo), Ch. Leitz (Universität Tübingen), S. Ortisi (Universität München),

E. Rova (Università Ca' Foscari Venezia), G. Ruffini (Fairfield University),

A. Sasson (San Diego Natural History Museum), H. Satzinger (Universität Wien),

S.M. Schellinger (The Ohio State University, Columbus), G. Schreiber (Eötvös Loránd University, Budapest),

E. Teeter (University of Chicago), S. Torallas-Tovar (University of Chicago),

Y. Tristant (Macquarie University, Sydney), V. Vaelske (independent researcher),

H. Vymazalová (Charles University, Prague), P. Weschenfelder (Universität Wien),

B. Williams (University of Chicago/PCMA UW, Warszawa),

K. Winther-Jacobsen (The Danish Institute at Athens), E. Zacharopoulou (University of Johannesburg),

P. Grotowski (UPJPII, Kraków), E. Papuci-Władyka, J. Śliwa (IA JU, Kraków), A. Ćwiek (IA AMU, Poznań),

K.O. Kuraszkiewicz, M. Pinker (FOS UW, Warszawa), Ł. Niesiołowski-Spanò (IH UW, Warszawa),

M. Gawlikowski, Mahmoud El-Tayeb (PCMA UW, Warszawa), S. Rzepka (IA UW, Warszawa)

\author{
RÉDACTION TECHNIQUE \\ Monika Wesołowska
}

REVUE DES TEXTES ANGLAIS

Jo Harper 
ÉTUDES et TRAVAUX

XXXII 
INSTYTUT KULTUR ŚRÓDZIEMNOMORSKICH I ORIENTALNYCH POLSKIEJ AKADEMII NAUK

\title{
STUDIA i PRACE
}

\author{
XXXII
}

gू IKŚiO

ESAN

WARSZAWA

2019 
INSTITUT DES CULTURES MÉDITERRANÉENNES ET ORIENTALES DE L’ACADÉMIE POLONAISE DES SCIENCES

\section{ÉTUDES et TRAVAUX}

XXXII

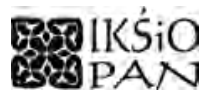

VARSOVIE

2019 
Publication scientifique financée dans le cadre du programme du Ministre de la Science et de l’Éducation Supérieure

« Programme National de Développement de l’Humanistique » pour les années 2016-2021 (projet no $3 b H 150099$ 83)

\title{
TI NARODOWY PROGRAM ROZWOJU HUMANISTYKI
}

\author{
Copyright $(\mathbb{C}$ \\ Instytut Kultur Śródziemnomorskich i Orientalnych PAN \\ et les Auteurs \\ Warszawa 2019
}

\begin{abstract}
ISSN 2084-6762
(avant 2011 : 0079-3566)

e-ISSN 2449-9579

Version première en papier, imprimée en Pologne - 150 copies

Version électronique accessible sur

http://www.etudesettravaux.iksiopan.pl
\end{abstract}

Édition: Polskie Towarzystwo Historyczne et Wydawnictwo Neriton, Warszawa

Conception générale de couverture : J. Iwaszczuk

Photo de couverture : P. Ligier ; courtoisie du Musée National de Varsovie

(Saint Jean Chrysostome, peinture, cathédrale de Faras). 


\section{Table des matières}

Editorial: Quid novi ex Nubia (par Bogdan Żurawski) ............................................... 7

Bibliography of Professor Stefan Jakobielski ................................................................ 13

Aneta Cedro, Bogdan Żurawski

Living with the Past in Modern Sudanese Village. Traditional Pottery Production

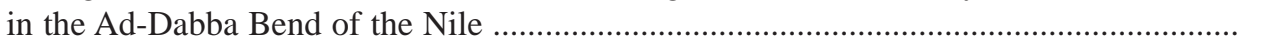

DAVID N. EDWARDS

A Possible Monastery and the 'Upper Maqs’ at Ukma-Akasha West? ............................ 53

Vincent W.J. van GeRven OeI

An Old Nubian Curse from the Faras Cathedral ........................................................ 81

Vincent W.J. van Gerven Oei, Adam Łajtar

Two Old Nubian Inscriptions from Akasha West ........................................................ 89

Vincent W.J. van Gerven Oei, Alexandros Tsakos

Rubrication Patterns in Two Old Nubian Manuscripts from Serra East .......................... 99

KAREL C. INNEMÉE

A Man in a Vessel, Once More .................................................................................. 111

KAREL C. INNEMÉE, Dobrochna ZielińSKA

Faces of Evil in Nubian Wall-Painting - An Overview ................................................ 121

ADAM ŁAJTAR

Epitaph of Merki Found in Hambukol ....................................................................... 145

MAGDALENA ŁAPTAŚ

Attributes, Vestments, Context and Inscription in the Identification of Nubian

Paintings: Proposing the 'Multi-Layer' Image Recognition Method ............................... 161

Grzegorz Ochala

Nubica Onomastica Miscellanea I: Notes on and Corrections to Personal Names

Found in Inscriptions from Faras 


\section{Robin SEIGNOBOS}

L'influence du Kitāb ahbār al-Nūba d'Ibn Sulaym al-Uswānī sur l'Histoire des églises et monastères d'Égypte d'Abū al-Makārim

JACQUES VAN DER VLIET

Exit Bishop Tamer - the Sequel. A New Edition of the Epitaph of Papsine alias Doulista (DBMNT 78) 